\title{
Mesoporous Titania Nanocrystals by Hydrothermal Template Growth
}

\author{
Giuseppe Cappelletti, Silvia Ardizzone, Francesca Spadavecchia, \\ Daniela Meroni, and Iolanda Biraghi \\ Dipartimento di Chimica Fisica ed Elettrochimica, Università degli Studi di Milano, Via Golgi 19, 20133 Milano, Italy \\ Correspondence should be addressed to Giuseppe Cappelletti, giuseppe.cappelletti@unimi.it
}

Received 6 May 2010; Revised 30 June 2010; Accepted 22 July 2010

Academic Editor: William W. Yu

Copyright (C) 2011 Giuseppe Cappelletti et al. This is an open access article distributed under the Creative Commons Attribution License, which permits unrestricted use, distribution, and reproduction in any medium, provided the original work is properly cited.

\begin{abstract}
Mesoporous $\mathrm{TiO}_{2}$ nanocrystals have been synthetized by a classical sol-gel route integrated by an hydrothermal growth step using monomeric (dodecylpyridinium chloride, DPC) or dimeric gemini-like (GS3) surfactants as template directing agents. Adsorption isotherms at the solid/liquid interface of the two surfactants have been obtained on aqueous dispersion of titania; the nature of the oxide/adsorbate interactions and the molecules orientation/coarea are discussed. The effects produced by the presence of the two surfactants on the different morphological (surface area, porosity, and shape) and structural (phase composition and aggregate size) features of the final $\mathrm{TiO}_{2}$ samples, calcined at $600^{\circ} \mathrm{C}$, are discussed.
\end{abstract}

\section{Introduction}

In recent years, the achievement of mesoporous titania nanomaterials, characterized by desired grain size, specific shape, high surface area, and tailored pore network, has became fundamental for photocatalytic and photovoltaic applications [1-4].

Several liquid-crystal templating (LCT) techniques, exploiting three-dimensional structures of ionic/nonionic surfactants [5-10] and block copolymers [11-13] have been proposed to synthesize mesoporous titanium oxides. The presence of mesoscopic pores in $\mathrm{TiO}_{2}$ for dye-sensitized solar cells is of great importance; in fact when the pores are filled with a conducting medium, charge carriers can easily percolate across the mesoscopic particle network making the whole internal surface area electronically accessible [14]. However, the fine control of the, often divergent, features of the material is very complex and the presence of the organic templating surfactant with the inherent high decomposition temperature may imply undesired effects. Just for these reasons, the extension of LCT techniques to pure titanium oxide has still remained elusive; the fast grain growth of the oxide, which occurs upon calcination of the surfactant-rich precursor, provokes, in fact, pore collapse with degradation of the mesostructure.

Besides the possible promotion of a controlled pore network, the presence of an amphiphilic molecule can intervene in different ways by tuning the sample final morphology. Very interesting is, for instance, the recent work by Chen et al. [16], where three-dimensionally ordered arrays of mesoporous titania spheres have successfully been synthesized through opal (ordered closed-packed face-centered cubic lattice of silica or latex spheres) template and triblock copolymer (Pluronic P123) as a mesopore-directing agent. The mean diameter of the titania spheres has been observed to be $c a .165 \mathrm{~nm}$ with a narrow distribution of the mesopore size in the range of $2-6 \mathrm{~nm}$.

Together with the surface area/porosity, also the grain size and relative enrichment in the different oxide polymorphs should be controlled through template routes to promote the best efficiency of the $\mathrm{TiO}_{2}$-based semiconductors $[9,10]$. Crystallite sizes in the range of $10-80 \mathrm{~nm}$ are considered optimal for both optical absorption and electron transport in the conversion of solar light to electric current, by dye-sensitized solar cells $[14,17,18]$. Anatase exhibits the highest activity for photovoltaic applications, while a 
controlled mixture of anatase and rutile or brookite is reported to produce the best activity in photodegradation experiments $[19,20]$. Mohamed et al. [5] reported that an hydrothermal treatment in the presence of cationic surfactants (cetyltrimethylammonium bromide, CTAB and cetylpyridinum bromide, $\mathrm{CPB}$ ) as templates may control not only the surface properties (surface area of $240-418 \mathrm{~m}^{2} \mathrm{~g}^{-1}$, pore size in the range of 23.2-43.7 $\AA$ ) and morphology (from nano-sized spheres to cotton fibrils) but also the bulk features (crystallites size in the range of 10.1-18.2 nm) and phase composition (rutile phase at $350^{\circ} \mathrm{C}$ ).

Here we report on the synthesis, of $\mathrm{TiO}_{2}$ nanocrystals, through a simple process implying a sol-gel starting reaction, followed by a growth step in solution, performed, also, in the presence of monomeric or dimeric "gemini-like" alkylpyridinium surfactants. The latter ones are composed by two conventional single-tail amphiphilic moieties chemically connected by spacer groups, usually polymethylene or short poly(oxyethylene) chains [10,21]. Recently, many studies concerning the practical use of gemini molecules appeared in the literature [22-25], showing that the first speculative interests were followed by practical applications, due to their unusual properties, such as the lower critical micellar concentration (CMC) values, the better adsorption behavior at both the air/water and the solid/water interfaces with respect to their single monomers, and the tendency to form micelles of different shapes and dimensions (i.e., spherical, rodlike, threadlike, vesicles), even at low concentration, when compared with similar nongemini surfactants. In this work, the concentration of surfactants in the ageing solution is varied in order to produce different conditions of selfaggregation between the surfactant molecules themselves. The surfactant/oxide interactions at the solid/liquid interface are evaluated by adsorption isotherms on aqueous suspension of titanium dioxide. The features of the calcined $\left(600^{\circ} \mathrm{C}\right)$ powders are investigated with respect to the phase composition/crystallinity, the surface area/porosity, and morphological aspects to evidence the effects provoked by the conditions of the particle template growth.

\section{Experimental Section}

All the chemicals were of reagent grade purity and were used without further purification; bi-distilled water passed through a Milli-Q apparatus was used to prepare solutions and suspensions.

2.1. Sample Preparation. $\mathrm{TiO}_{2}$ particles were obtained by following a room-temperature sol-gel reaction starting from $\mathrm{Ti}\left(\mathrm{C}_{4} \mathrm{H}_{9} \mathrm{O}\right)_{4}$ and adopting a water/alkoxide molar ratio of 81.7 and a water/propanol molar ratio of 8.5. The xerogel powders were purified by centrifugation-resuspension cycles and then powder fractions were aged at $80^{\circ} \mathrm{C}$, at $\mathrm{pH} 8$ (i.e., condition of attractive electrostatic interactions between the oxide and the cationic surfactants), for fixed timelength $(5 \mathrm{~h})$ with different surfactants concentrations: $1-100 \mathrm{mM}$ and $0.1-50 \mathrm{mM}$ for dodecylpyridinium chloride, DPC (compound 1) and 1, 1' -didodecil-2, 2' -trimetilendipiridinio-dicloruro, "gemini spacer 3" GS3 (compound 2), respectively.
After the ageing, the suspensions were dried again at $80^{\circ} \mathrm{C}$. Finally the powders were thermally treated at $600^{\circ} \mathrm{C}$ for 6 hours under an oxygen stream.

2.2. Sample Characterization. Room-temperature X-ray powder diffraction (XRPD) patterns were collected between 10 and $80^{\circ}$ with a Siemens D500 diffractometer, using $\mathrm{Cu}$ $\mathrm{K}_{\alpha}$ radiation. Rietveld refinement has been performed using the GSAS software suite and its graphical interface EXPGUI $[26,27]$. The average diameter of the crystallites, $\langle\mathrm{D}\rangle$, was estimated from the most intense reflection of the anatase (101) and rutile (110) $\mathrm{TiO}_{2}$ phases using the Scherrer equation [28].

Specific surface areas were determined by the classical BET procedure using a Coulter SA 3100 apparatus. Desorption isotherms were used to determine the pore size distribution using the Barret-Joyner-Halander (BJH) method.

Scanning electron microscopy (SEM) photographs are acquired with a LEO 1430.

The values of the critical micelle concentration (CMC) of DPC and gemini surfactants were obtained by conductometric determinations as a function of the temperature. Therefore, using as a guideline an average increasing slope with the temperature of the CMC of DPC and GS3, obtained from the present results and literature data [15, 21, 29-32], a concentration range roughly corresponding to a possible $\mathrm{CMC}$ at $80^{\circ} \mathrm{C}$ was estimated for both surfactants $(18-22 \mathrm{mM}$, for DPC and 2-4 mM in the case of GS3).

The adsorption isotherm of GS3 at the $\mathrm{TiO}_{2}$ interface was obtained under the following conditions: $T=25 \pm 0.3^{\circ} \mathrm{C}$; $\mathrm{pH}=8.0 \pm 0.2$; equilibration time $=4 \mathrm{~h}$; ionic strength $I=2 \times 10^{-3} \mathrm{M} \mathrm{KCl}$. At the end of the adsorption time, the surnatant solution was sampled for the residual surfactant concentration by spectrophotometric characterization at $265 \mathrm{~nm}$ for the gemini salt. Data on DPC adsorption on $\mathrm{TiO}_{2}$, obtained by Koopal et al. [15] are elaborated and reported for comparison $\left(T=21 \pm 1^{\circ} \mathrm{C} ; \mathrm{pH}=8.0 \pm 0.2\right.$; equilibration time $=12 \mathrm{~h}$; ionic strength $I=1 \times 10^{-3} \mathrm{M}$ $\mathrm{NaCl}$ ). Adsorption isotherms of the two surfactant molecules are reported by plotting the surface excess $(\Gamma)$ as a function of the final concentration at equilibrium.

\section{Results and Discussion}

3.1. Adsorption at $\mathrm{TiO}_{2}$-Solution Interface. Figure 1 reports the experimental adsorption isotherms, at the $\mathrm{TiO}_{2}$ solution interface, of both monomeric DPC (Figure 1(a), elaborated from data in [15]) and dimeric "gemini-like" GS3 (Figure 1(b)) surfactants. The shape of the two curves is markedly different. The monomer isotherm (Figure 1(a)) is S-shaped showing a low adsorbent-adsorbate affinity due to weak interactions between the surfactant and the oxide [15]; moreover, it can be proposed that the adsorption leads to the formation of surface ion pairs, $\mathrm{Ti}-\mathrm{O}^{-} \mathrm{Py}^{+}$(the surface charge of $\mathrm{TiO}_{2}$ at $\mathrm{pH} 8$ is negative), provoking a partial compensation of the surfactant aromatic charge, in accordance with our previous XPS results [10]. The curve pertaining to the gemini (Figure 1(b)), instead, follows the trend classified in the literature as L-type, which is generally 


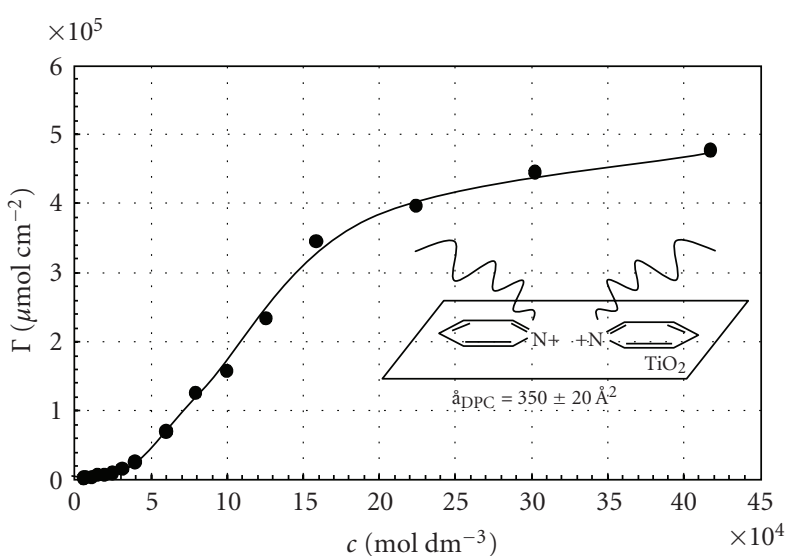

(a) $\mathrm{DPC}-\mathrm{TiO} 2$

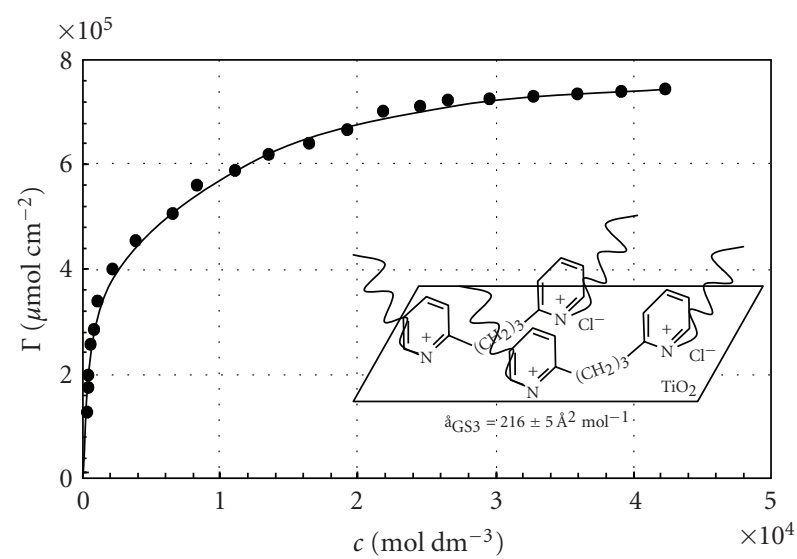

(b) $\mathrm{GS} 3-\mathrm{TiO}_{2}$

FIgURE 1: Adsorption isotherms (surface excess versus equilibrium concentrations) at the $\mathrm{TiO}_{2} /$ solution interface of (a) monomeric DPC and (b) dimeric GS3 surfactants. Inset: sketches of possible disposition/orientations of the adsorbed surfactant at the titania surface; $\stackrel{\circ}{\text { is }}$ the adsorbate coarea value, calculated from the maximum coverage $\left(\Gamma_{\max }\right)$. Data of DPC are reelaborated from [15].

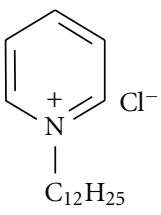

2. DPC

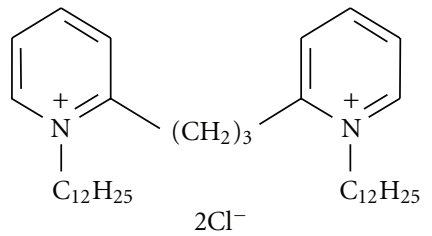

1. GS3
TABLe 1: Data ( $\beta$, adsorption equilibrium constant; $\triangle G_{0}$, standard adsorption Gibbs energy; $a$, lateral interaction parameter) from the elaboration of GS3 and DPC isotherms.

\begin{tabular}{lccccc}
\hline isotherm & model & $R^{2}$ & $\beta$ & $\triangle G^{0} \mathrm{~kJ} \mathrm{~mol}^{-1}$ & $a$ \\
\hline DPC & FFG & 0.97 & $160 \pm 20$ & $-5.6 \pm 0.2$ & $3.2 \pm 0.2$ \\
GS3 & Langmuir & 0.997 & $48000 \pm 6000$ & $-27.1 \pm 0.3$ & -
\end{tabular}

Scheme 1

associated with coverage of the solid surface by a monolayer of adsorbate molecules and is characterized by a strong adsorbent-adsorbate interaction. The higher affinity of the gemini GS3 for the solid appears also from the larger adsorbed amounts $\left(\Gamma_{\max }=7.7 \times 10^{-5} \mu \mathrm{mol} \mathrm{cm}{ }^{-2}\right)$ in the quasiplateau regions with respect to the maximum surface excess $\left(\Gamma_{\max }=4.8 \times 10^{-5} \mu \mathrm{mol} \mathrm{cm}{ }^{-2}\right)$ of the monomeric DPC surfactant. For these reasons, the data relative to the DPC and GS3 isotherms were elaborated on the basis of the Langmuir (strong adsorbent-adsorbate interaction, no lateral interactions between adsorbate molecules) and the Frumkin-Fowler-Guggenheim (FFG, weak adsorbentadsorbate interaction, electrostatic and nonelectrostatic lateral interactions [33-35]) model equations, respectively. The parameters obtained from the treatment of the data are reported in Table 1. The linear correlation $\left(R^{2}\right)$ is good in both cases, justifying the choice of the two different model approaches. The adsorption equilibrium constants $(\beta)$ and the relative standard adsorption Gibbs energy $\left(\triangle G^{0}\right)$ values support the dramatic increase in adsorption at the $\mathrm{TiO}_{2}$ surface passing from the monomer to the dimer [36]. In the case of the DPC isotherm, the lateral interaction parameter (a) is positive, that is, it represents electrostatic repulsion interactions between the positive charges of the surfactant headgroups, in agreement with what reported by Mehrian et al. [37] in the case of DPC adsorption on clays. They studied the influence of the electrolyte concentration on the adsorption of DPC on Na-kaolinite; an attractive lateral term was obtained only in the case of high ionic strength $(100 \mathrm{mM})$, while repulsion prevailed at lower electrolyte concentrations (5 and $20 \mathrm{mM}$ ).

The limiting areas ( $\stackrel{a}{a}$ co-area) for DPC and GS3 (350 and $216 \AA^{2}$, resp.) at maximum packing $\left(\Gamma_{\max }\right)$ calculated from the surface excess at maximum coverage are higher than those present in the literature. Ottewill et al. [38] reported two different values of coarea for DPC molecule, obtained by surface tension at the air-water interface, in the case of flat-lying $\left(110 \AA^{2}\right)$ and vertical $\left(35 \AA^{2}\right)$ orientations. Thus, on the basis of these considerations, the present size of adsorbed DPC molecule could be interpreted as being due to a flat orientation (sketch inset Figure 1), with the formation of a diluted film, characterized by electrostatic repulsion between the charged pyridinium heads. On the contrary, the lower value of $a$ for the GS3 surfactant could be ascribable to a vertical orientation (see sketch inset Figure 1) in which, in agreement with our previous results of adsorption of gemini zero-spacer on $\mathrm{TiO}_{2}$ [36], only one pyridinic group is directly involved in the electrostatic interactions with the oxide, whereas the second one is compensated by its counterion $\left(\mathrm{Cl}^{-}\right)$.

Further information concerning the surfactant adsorption isotherms can be appreciated from the log-log coordinates (Figure 2). The primary advantage of using a loglog plot is that it amplifies the features of the isotherm at low surface excess values. The general form of isotherms plotted in this manner and the morphology of adsorbed 


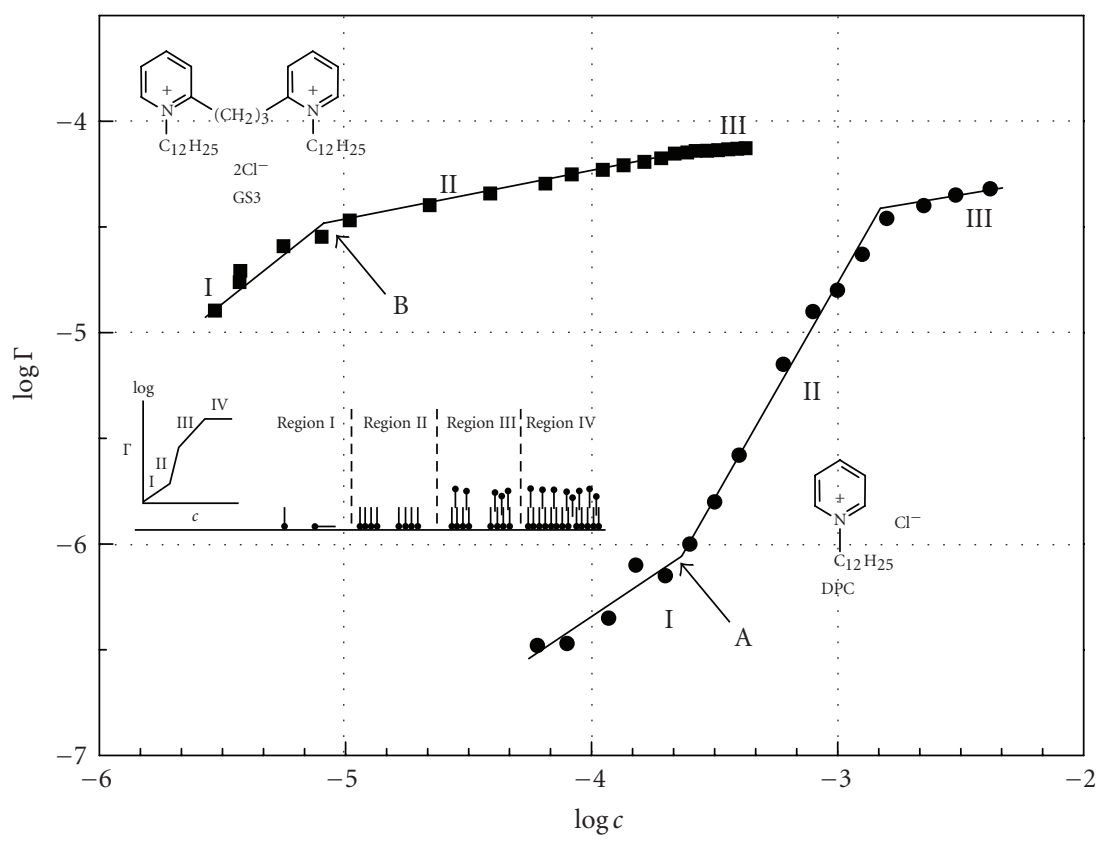

FIGURE 2: Log-log plot of the surface excess $(\Gamma)$ as a function of the equilibrium concentration for DPC and GS3 surfactants. Inset: the general shape of surfactants adsorption isotherms at solid substrates and the proposed four-region model of adsorption.

structures associated with each region are depicted schematically in the inset of Figure 2. Somasundaran et al. [39, 40] proposed the four-region model attributed, respectively, to the adsorption of (I) monomers by electrostatic interactions, (II) surface aggregates (hemimicelles) up to the substratum charge compensation, (III) headout molecules by chainchain interactions, and (IV) fully formed bilayer. Further increases in the solution surfactant concentration do not lead to any further increases in the surface excess. From the plot (Figure 2), only three regions are appreciable since the isotherms are limited to monolayer coverage (for both surfactants, $c_{\text {SURF }}<\mathrm{CMC}$ ) without the complete formation of bilayers. The surfactant concentration relative to the beginning of hemimicelle formation is about $1.8 \times 10^{-4} \mathrm{M}$ and $8.0 \times 10^{-6} \mathrm{M}$, respectively for DPC (A point) and GS3 (B point); these values could be compared with the bulk CMC values (DPC: $1.6 \times 10^{-2} \mathrm{M}, \mathrm{GS} 3: 1.5 \times 10^{-3} \mathrm{M}$ at $25^{\circ} \mathrm{C}$ ) obtained from conductimetric/tensiometric determinations $[15,21,29-32]$, following the criterion reported by Fuerstenau and Jang [41] that hemimicelles may occur in about $1 / 100$ th of the CMC.

The trend between the two isotherms (the shift along the $x$-axis, the variation of the slope in the different regions and the distribution of surface excesses) is the expected one due to the presence of a second homologous pyridinic ring in the case of GS3 species. For both surfactants, the slope of the plot in the Region I (the Henry region) is unity in the ideal situation $\left(\triangle G^{0}\right.$ is constant) [15]. The initial slope of the experimental isotherms cannot be determined accurately, but they are, within experimental error, almost equal to unity. The slope of the DPC curve increases passing from Region I to Region II, where hemimicelles form, indicating that the adsorption is enhanced by the formation of surface aggregates. This trend is typical for S-shaped isotherms [15]. In the case of the gemini molecules the slope is, instead, depressed in the region of hemimicelles, possibly due to the steric hindrance.

3.2. Self-Aggregation Features of DPC and GS3 Surfactants. It is well known that many physicochemical properties of a solution show sudden changes when a characteristic concentration of a surfactant is exceeded. This change is attributed to the formation of colloidal aggregates and occurs over a relatively small concentration range characteristic for the surfactant. The formation of direct micelles of DPC in water and in aqueous salt solutions has been the object of numerous studies performed in time by different experimental approaches. By scattering techniques, DPC is reported to form in water, globular micelles with aggregation number of about 20 , with a corresponding micellar radius of about $1.9 \mathrm{~nm}$, comparable with the extended length of a $\mathrm{C}_{12}$ chain $(1.67 \mathrm{~nm})[30-32,42,43]$. Spherical DPC micelles are not reported to aggregate in tridimensional structures [31]. The corresponding gemini surfactant (GS3) having short spacer $(n=3)$ has a particular behavior since it binds its counterions more strongly than the "single" does, as the $n-s-n$ ammonium gemini amphiphiles [44-47]. The lack of conformational freedom could be the result of the sharing of one counterion between the two pyridinium head groups [21]. As a consequence, the short spacer surfactant (GS3) is probably aggregating in nonspherical micelles, but in elongated forms, like rods, as reported in the literature for ammonium gemini surfactants [48-50]. In particular, Manne et al. [48] found that simmetric gemini C12-sC12 $(s \leq 6)$ surfactants composed by linear hydrocarbon tail with quaternary ammonium headgroups may assemble 


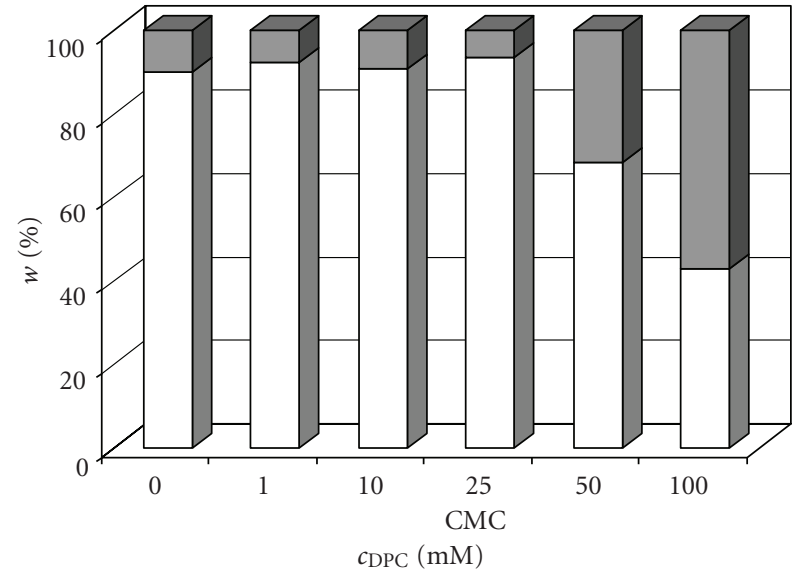

(a)

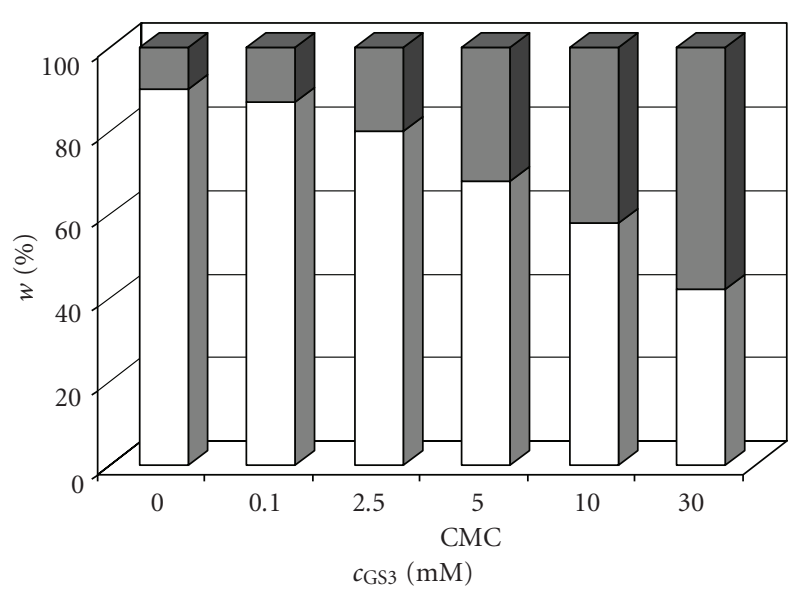

(b)

FIGURE 3: Relative phase enrichment in anatase $(\square)$ and rutile $\mathrm{TiO}_{2}$ polymorphs of samples grown in surfactant solutions and subsequently calcined at $600^{\circ} \mathrm{C}$. (a) DPC, (b) GS3.

in hexagonal cylinders in silicate mesophase and parallel cylinders at the mica surface with average spacings of $4.2 \pm$ $0.4 \mathrm{~nm}$. Thus, possibly cylinders in hexagonal arrangement for gemini spacer 3 could also occur at high surfactant concentrations.

\subsection{Structural and Morphological Results of Surfactant-} Assisted Titania Samples. Recently a great interest was devoted to the growth of titania particles with controlled morphology and porosity through template and templatefree procedures [51-53]. Yu et al. obtained by a templatefree preparation of $\mathrm{TiO}_{2}$ a fine control of macro and mesoporosity up to about $500^{\circ} \mathrm{C}$. For higher calcination temperatures, a total collapse of both surface area and porosity took place.

In the present work, the effects provoked by the concentration of both monomeric (DPC) and gemini (GS3) alkylpyridinium salts in the growth solution, on the features of the $\mathrm{TiO}_{2}$ particles are considered. Figure 3 reports the
TABLE 2: Anatase (A), rutile (R) domain sizes and BET surface area for all samples.

\begin{tabular}{lllll}
\hline \multicolumn{2}{c}{ Sample } & $\left\langle D_{\mathrm{A}}\right\rangle(\mathrm{nm})$ & $\left\langle D_{\mathrm{R}}\right\rangle(\mathrm{nm})$ & $\begin{array}{l}S_{\mathrm{BET}} \\
\left(\mathrm{m}^{2} \mathrm{~g}^{-1}\right)\end{array}$ \\
\hline \multirow{4}{*}{ no SURF } & 28 & - & 18 \\
& $1 \mathrm{mM}$ & 20 & - & 19 \\
$\mathrm{DPC}$ & $10 \mathrm{mM}$ & 22 & - & 19 \\
& $25 \mathrm{mM}$ & 24 & - & 16 \\
& $(\sim \mathrm{CMC})$ & & & 8 \\
& $50 \mathrm{mM}$ & 32 & 86 & 5 \\
\hline \multirow{4}{*}{ GS3 } & $100 \mathrm{mM}$ & 41 & $>100$ & 20 \\
& $0.1 \mathrm{mM}$ & 23 & - & 16 \\
& $2.5 \mathrm{mM}$ & 30 & 54 & 13 \\
& $5 \mathrm{mM}$ & 37 & 63 & 14 \\
& $(\sim \mathrm{CMC})$ & & 73 & 11 \\
\hline
\end{tabular}

relative phase enrichment (histograms) at different surfactant concentrations of samples submitted to a hydrothermal growth step in the presence of the DPC and GS3, subsequently calcined at $600^{\circ} \mathrm{C}$. The anatase and rutile amount in the samples appears to depend on the concentration of the surfactant in the solution adopted for the ageing. When the concentration corresponds to the existence of nonaggregated surfactant units $(1,10 \mathrm{mM}$ for DPC and $1,2.5 \mathrm{mM}$ for GS3), the anatase polymorph is prevalent; the crystallite sizes (Table 2) appear to be smaller than those in the absence of surfactant. The specific surface areas of the calcined oxide remain quite the same (Table 2). By increasing the surfactant concentration at values larger than the CMC $(>25 \mathrm{mM}$ for DPC and $>5 \mathrm{mM}$ for GS3), the amount of anatase decreases and the anatase and rutile structures are almost equally promoted. The formation of rutile is paralleled by larger crystallite sizes and lower surface areas (Table 2). Moreover, the presence of GS3 at higher concentrations seems to preserve the surface area $\left(11-14 \mathrm{~m}^{2} \mathrm{~g}^{-1}\right)$ and to limit the increase in rutile crystallite sizes $(\sim 75 \mathrm{~nm})$ with respect to the DPC surfactant $\left(5-8 \mathrm{~m}^{2} \mathrm{~g}^{-1}\right.$ and $>85 \mathrm{~nm}$, resp.).

These results are considered to be significant since after a calcination at $600^{\circ} \mathrm{C}$ both the surface area and the total pore volume are still appreciable at variance with samples prepared by template-free procedures [51-53]. The trend in Figure 3 is in agreement with the generally reported lower surface energy of anatase with respect to rutile $[54,55]$ and with the consistent finding that the phase transformation to rutile occurs after the anatase grains have grown to a certain threshold size, of about 30-40 nm [56]. Actually, during the hydrothermal step particle growth through Ostwald ripening can be expected to occur [57]. When the growth occurs in the presence of a non-aggregated surfactant, the surface of the precursor particles is shielded from the deposition of soluble $\mathrm{Ti}$ species by the presence of the surfactant film. The growth is slightly depressed, the particles remain smaller and stable supporting the formation of anatase. At higher 


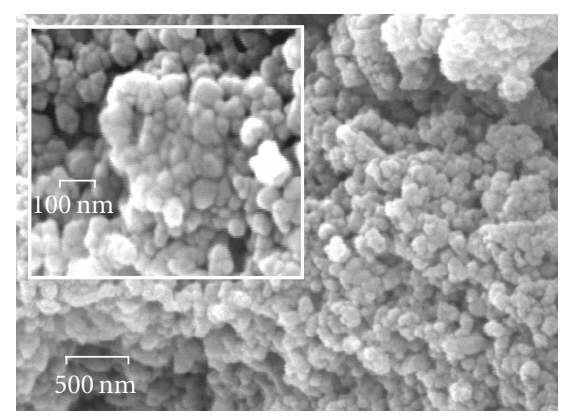

(a)

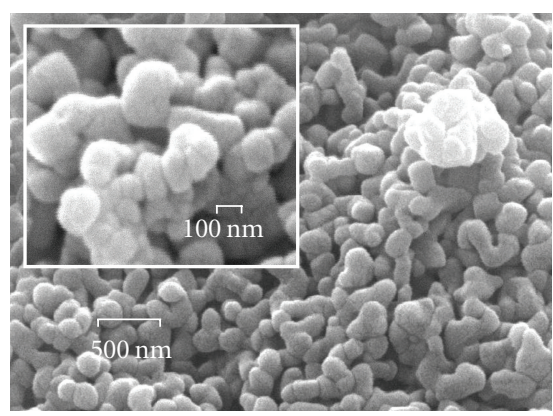

(b)

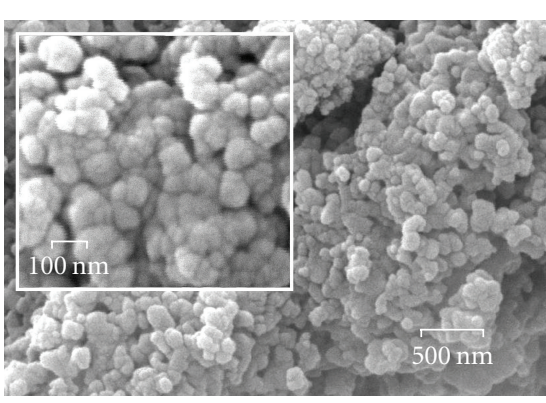

(c)

FIGURE 4: SEM micrographs of titania particles hydrothermally grown in absence of surfactant (a) and in presence of high concentration of (b) DPC (100 mM) and (c) GS3 $(30 \mathrm{mM})$, subsequently calcined at $600^{\circ} \mathrm{C}$.

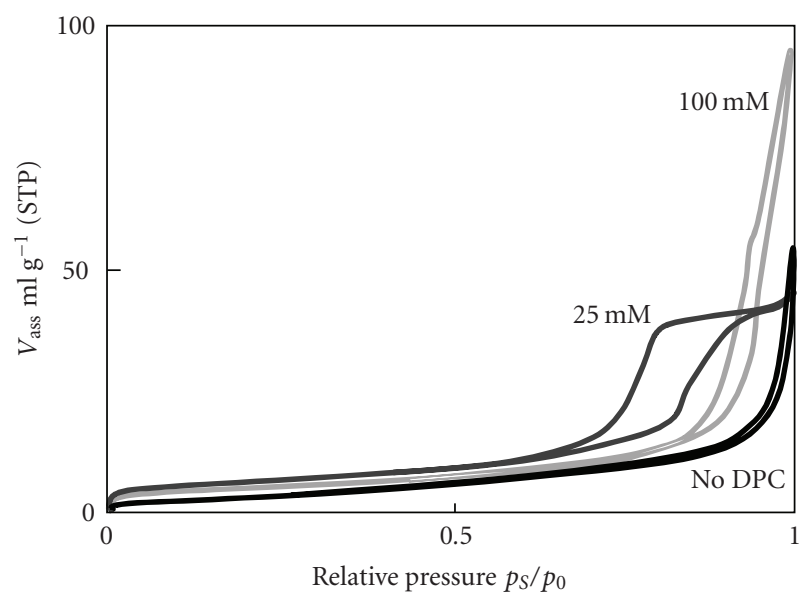

(a)

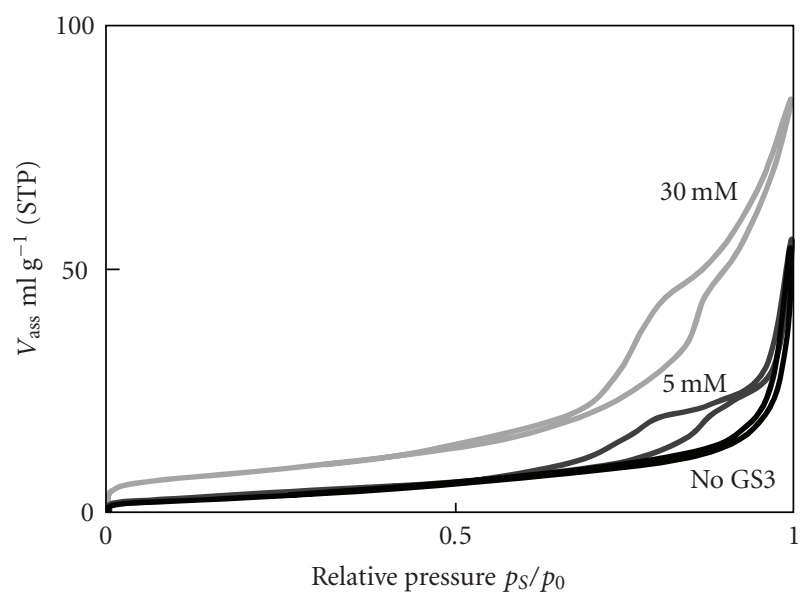

(b)

FIgURE 5: $\mathrm{N}_{2}$ adsorption-desorption isotherms of $\mathrm{TiO}_{2}$ samples aged in different surfactant solutions and calcined at $600^{\circ} \mathrm{C}$. (a) DPC, (b) GS3

concentrations, the micelles may act as carriers for the soluble Ti containing species promoting the growth. Further, the micelles may be, also, adsorbed at the surface of different particles and may therefore promote bridging phenomena leading to larger particles.

These considerations are closely mirrored by the particle morphology as apparent in SEM micrographs (Figure 4). In the case of the sample prepared in the absence of the surfactant (Figure 4(a)), small spheroidal particles with an average size of about $30 \mathrm{~nm}$ grouped in raspberry-like aggregates can be appreciated; in the case instead of the sample grown at high DPC concentration (Figure $4 \mathrm{~b}$ ) the particles are much larger, with sizes ranging from about $100 \mathrm{~nm}$ (single crystals) and to about $200-300 \mathrm{~nm}$ as the result of sintering between crystallites. The large particles in Figure 4(b), show a particularly smooth surface. This effect is presumably the result of a surface annealing provoked by the heat released during the surfactant combustion. In the case of titania particles grown at high GS3 concentration, the shape of the aggregates remains spheroidal, characterized by a diameter of $\sim 60-70 \mathrm{~nm}$.
The mesoporosity induced by micelles and further threedimensional organization of both surfactants can be appreciated by the hysteresis loop of the nitrogen adsorptiondesorption isotherm (Figure 5), with respect to the reference material prepared without surfactant. The hydrothermal treatment in the presence of DPC micelles $\left(c_{\mathrm{DPC}}=25 \mathrm{mM}\right.$, Figure 5 (a)) leads to titania particles with bottle-neck shaped pores mainly in the range of $6<d<10 \mathrm{~nm}$ (Figure 6 (a)), comparable with the average diameter of the globular DPC micelles $(\sim 4 \mathrm{~nm})$ [48]. When the particles are grown at $c_{\mathrm{DPC}}>\mathrm{CMC}$, the shape of hysteresis loops is similar to the reference sample; the total pore volume increases, but the fraction of desired mesopores is not relevant. On the other hand, the presence of GS3 cylindrical micelles and three-dimensional hexagonal arrangements (sketches in Figure 6 (b)) produces an increase of total pore volume at increasing surfactant concentration (Figure 6 (b)), especially the fraction of mesopores with diameters in the range 6 $<d<20 \mathrm{~nm}$. The shape of the hysteresis loop is typical of open-ended slit-shaped pores (Figure 5 (a)). A typical pore size distribution curve for the hydrothermally treated 

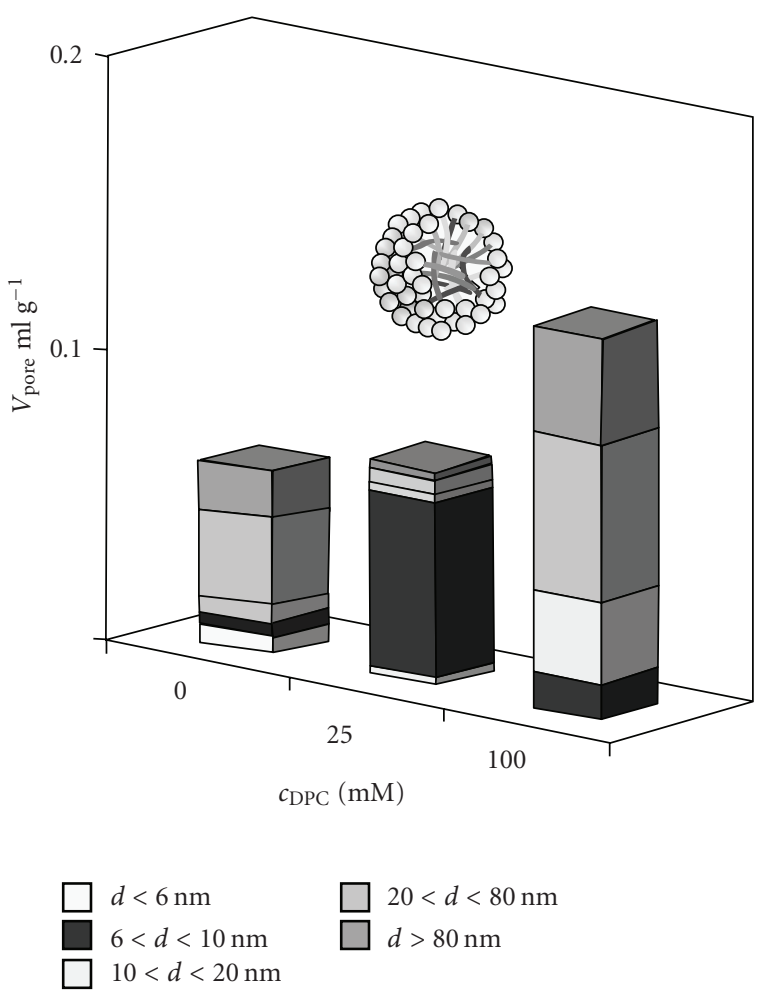

(a)

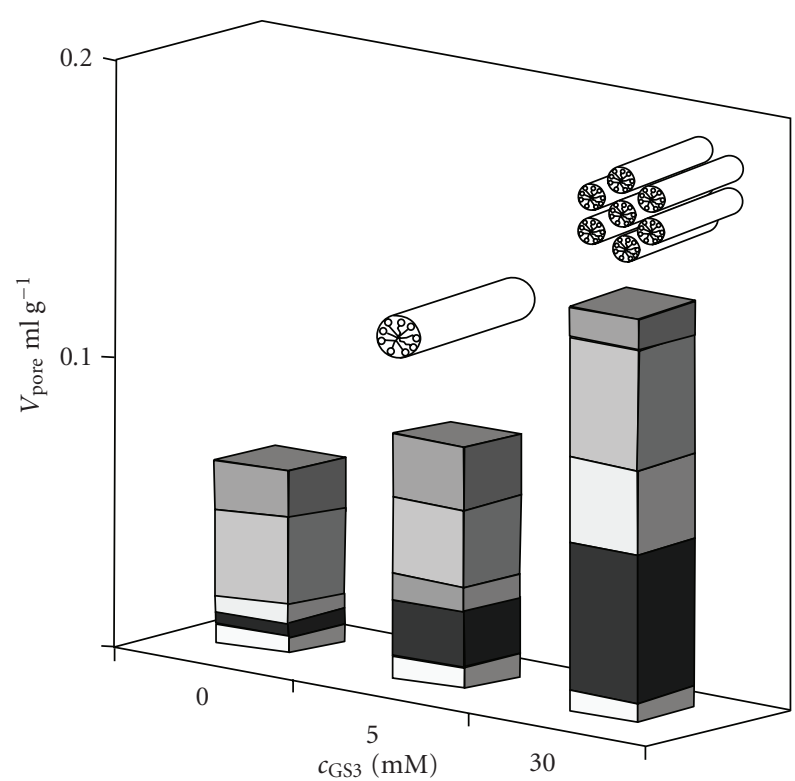

$\square d<6 \mathrm{~nm}$
$\square 6<d<10 \mathrm{~nm}$
$\square 10<d<20 \mathrm{~nm}$

(b)

Figure 6: Pore volume distribution of $\mathrm{TiO}_{2}$ samples after (a) DPC and (b) GS3 ageing as a function of surfactant concentration and calcined at $600^{\circ} \mathrm{C}$. Insets: possible surfactant three-dimensional aggregations.

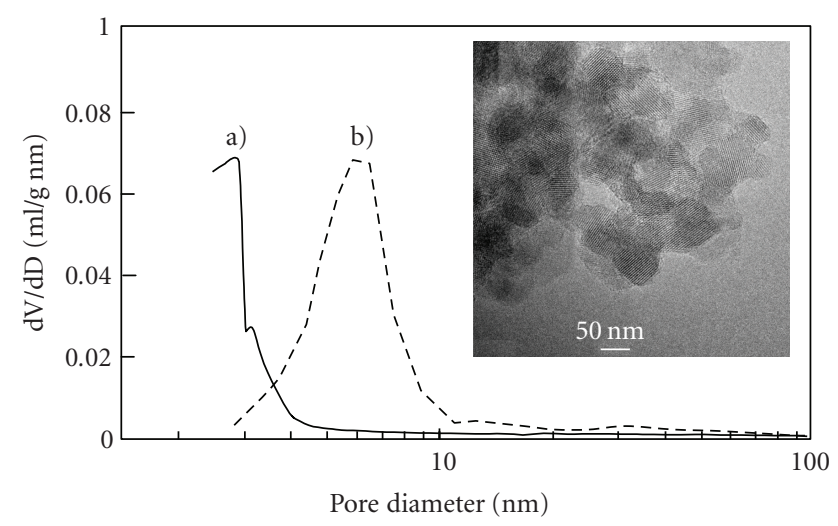

Figure 7: Pore size distribution curve of (a) $\mathrm{TiO}_{2}$ bare particles and (b) after GS3 ageing $(30 \mathrm{mM})$ calcined at $600^{\circ} \mathrm{C}$. Inset: HRTEM image of GS3 hydrothermally treated and calcined sample.

$\mathrm{TiO}_{2}$ in the presence of three-dimensional GS3 hexagonal arrangements is shown in Figure 7. A fairly narrow size distribution, $6-20 \mathrm{~nm}$, is achieved for the present sample at variance with the untreated one (calcined at $600^{\circ} \mathrm{C}$ ), which shows almost no porosity. This result indicates that the hydrothermal template treatment of the $\mathrm{TiO}_{2}$ particles leads to their aggregation with evolution of a mesoporosity probably arising from the template structure, which is successively thermally decomposed. Part of the mesoporous structure can be appreciated in the HRTEM image of inset of Figure 7.

These results, together with the surface and morphological aspects, could be explained by invoking the different local heat of combustion (during the calcination step) for DPC and GS3, especially for higher surfactant concentrations. Possible ordered hexagonal GS3 arrangements with respect to unstructured spherical DPC micelles with random size distribution may lead to lower surfactant/oxide ratio in the template growth, even if the GS3 surfactant is formed by double aromatic ring.

\section{Conclusion}

The features of the adsorption isotherms of monomeric (DPC) and gemini (GS3) alkylpyridinium surfactants at the $\mathrm{TiO}_{2}$ solution interface are markedly different. DPC gives rise to a very diluted surface film, characterized by weak adsorbate/adsorbent interactions and repulsive lateral interactions. This behaviour is paralleled by a weak selfaggregation tendency, apparent in the sole formation of very small, globular micelles and no long range ordered mesostructures. The behaviour of the gemini, instead, is typical of strong (chemi- and physi-) interactions with $\mathrm{TiO}_{2}$ occurring in the absence of lateral interactions. The vertical orientation of the molecule, suggested on the grounds of the 
present adsorption data, is fully consistent with the formation of elongated rods and further hexagonal arrangements proposed for gemini surfactant mesostructures.

The above-mentioned specific features of the two surfactants are actually the reason why they produce different effects on the final oxide characteristics, especially on its mesoporosity.

For growths performed at concentrations larger than the CMC, very numerous small DPC micelles are randomly embedded into the oxide, giving rise, during the ensuing calcinations step, to large heats of combustion, particle sintering, and an almost uncontrolled mesoporosity.

In the case of gemini, instead, the relevant surfactant/oxide interactions allow the templating function of the surfactant to significantly occur. The much lower surfactant/oxide ratio than in the case of DPC, inherent in the rods versus globule structure, leads to a lower heat of combustion and to controlled particle shape, size, and mesoporosity.

\section{Acknowledgments}

The authors wish to thank Professor G. Viscardi and Professor P. Quagliotto of Università di Torino for the synthesis of the gemini spacer 3 (GS3) surfactant. This research has been supported by the University of Milan Research Funds (FIRST, PUR).

\section{References}

[1] A. S. Attar, M. S. Ghamsari, F. Hajiesmaeilbaigi, SH. Mirdamadi, K. Katagiri, and K. Koumoto, "Sol-gel template synthesis and characterization of aligned anatase- $\mathrm{TiO}_{2}$ nanorod arrays with different diameter," Materials Chemistry and Physics, vol. 113, no. 2-3, pp. 856-860, 2009.

[2] H. Chen, J. Yan, Z. Ye et al., "One pot synthesis of mesostructured non-silica oxides nanocrystallites," Journal of Materials Science, vol. 44, no. 24, pp. 6531-6537, 2009.

[3] D. P. Wang and H. C. Zeng, "Nanocomposites of anatasepolyaniline prepared via self-assembly," Journal of Physical Chemistry C, vol. 113, no. 19, pp. 8097-8106, 2009.

[4] F. Spadavecchia, G. Cappelletti, S. Ardizzone et al., "Solar photoactivity of nano-N-TiO $\mathrm{T}_{2}$ from tertiary amine: role of defects and paramagnetic species," Applied Catalysis B, vol. 96, no. 3-4, pp. 314-322, 2010.

[5] M. M. Mohamed, W. A. Bayoumy, M. Khairy, and M. A. Mousa, "Synthesis of micro-mesoporous $\mathrm{TiO}_{2}$ materials assembled via cationic surfactants: morphology, thermal stability and surface acidity characteristics," Microporous and Mesoporous Materials, vol. 103, no. 1-3, pp. 174-183, 2007.

[6] A. Mitra, A. Bhaumik, and B. K. Paul, "Synthesis and characterization of mesoporous titanium dioxide using selfassembly of sodium dodecyl sulfate and benzyl alcohol systems as templates," Microporous and Mesoporous Materials, vol. 109, no. 1-3, pp. 66-72, 2008.

[7] A.-C. Lee, R.-H. Lin, C.-Y. Yang, M.-H. Lin, and W.-Y. Wang, "Preparations and characterization of novel photocatalysts with mesoporous titanium dioxide $\left(\mathrm{TiO}_{2}\right)$ via a sol-gel method," Materials Chemistry and Physics, vol. 109, no. 2-3, pp. 275-280, 2008.

[8] D. Chandra and A. Bhaumik, "Super-microporous $\mathrm{TiO}_{2}$ synthesized by using new designed chelating structure directing agents," Microporous and Mesoporous Materials, vol. 112, no. 1-3, pp. 533-541, 2008.

[9] G. Cappelletti, C. L. Bianchi, and S. Ardizzone, "XPS study of the surfactant film adsorbed onto growing titania nanoparticles," Applied Surface Science, vol. 253, no. 2, pp. 519-524, 2006.

[10] S. Ardizzone, C. L. Bianchi, and G. Cappelletti, "Growth of $\mathrm{TiO}_{2}$ nanocrystals in the presence of alkylpyridinium salts: the interplay between hydrophobic and hydrophilic interactions," Surface and Interface Analysis, vol. 38, no. 4, pp. 452-457, 2006.

[11] D. S. Kim, S. J. Han, and S.-Y. Kwak, "Synthesis and photocatalytic activity of mesoporous $\mathrm{TiO}_{2}$ with the surface area, crystallite size, and pore size," Journal of Colloid and Interface Science, vol. 316, no. 1, pp. 85-91, 2007.

[12] C. Tian, Z. Zhang, J. Hou, and N. Luo, "Surfactant/copolymer template hydrothermal synthesis of thermally stable, mesoporous $\mathrm{TiO}_{2}$ from $\mathrm{TiOSO}_{4}$," Materials Letters, vol. 62, no. 1, pp. 77-80, 2008.

[13] J. Procházka, L. Kavan, V. Shklover et al., "Multilayer films from templated $\mathrm{TiO}_{2}$ and structural changes during their thermal treatment," Chemistry of Materials, vol. 20, no. 9, pp. 2985-2993, 2008.

[14] M. Grätzel, "Photoelectrochemical cells," Nature, vol. 414, no. 6861, pp. 338-344, 2001.

[15] L. K. Koopal, E. M. Lee, and M. R. Böhmer, "Adsorption of cationic and anionic surfactants on charged metal oxide surfaces," Journal of Colloid and Interface Science, vol. 170, no. 1, pp. 85-97, 1995.

[16] J. Chen, Z. Hua, Y. Yan, A. A. Zakhidov, R. H. Baughman, and L. Xu, "Template synthesis of ordered arrays of mesoporous titania spheres," Chemical Communications, vol. 46, no. 11, pp. 1872-1874, 2010.

[17] M. Grätzel, "Mesoscopic solar cells for electricity and hydrogen production from sunlight," Chemistry Letters, vol. 34, no. 1, pp. 8-13, 2005.

[18] Q. Shen and T. Toyoda, "Studies of optical absorption and electron transport in nanocrystalline $\mathrm{TiO}_{2}$ electrodes," Thin Solid Films, vol. 438-439, pp. 167-170, 2003.

[19] D. C. Hurum, A. G. Agrios, K. A. Gray, T. Rajh, and M. C. Thurnauer, "Explaining the enhanced photocatalytic activity of Degussa P25 mixed-phase $\mathrm{TiO}_{2}$ using EPR," Journal of Physical Chemistry B, vol. 107, no. 19, pp. 4545-4549, 2003.

[20] R. Andreozzi, V. Caprio, A. Insola, and R. Marotta, "Advanced oxidation processes (AOP) for water purification and recovery," Catalysis Today, vol. 53, no. 1, pp. 51-59, 1999.

[21] P. Quagliotto, G. Viscardi, C. Barolo et al., "Gemini pyridinium surfactants: synthesis and conductometric study of a novel class of amphiphiles," Journal of Organic Chemistry, vol. 68, no. 20, pp. 7651-7660, 2003.

[22] S. K. Hait and S. P. Moulik, "Gemini surfactants: a distinct class of self-assembling molecules," Current Science, vol. 82, no. 9, pp. 1101-1111, 2002.

[23] R. Zana, "Gemini (dimeric) surfactants," Current Opinion in Colloid and Interface Science, vol. 1, no. 5, pp. 566-571, 1996.

[24] M. J. Rosen and D. J. Tracy, "Gemini surfactants," Journal of Surfactants and Detergents, vol. 1, no. 4, pp. 547-554, 1998.

[25] F. M. Menger and J. S. Keiper, "Gemini surfactants," Angewandte Chemie, vol. 39, no. 11, pp. 1906-1920, 2000.

[26] A. C. Larson and R. B. Von Dreele, "GSAS: General Structural Analysis System," Laboratory Report LAUR 86-748, Los Alamos National Laboratory, Los Alamos, NM, USA, 1994.

[27] B. H. Toby, "EXPGUI, a graphical user interface for GSAS," Journal of Applied Crystallography, vol. 34, no. 2, pp. 210-213, 2001. 
[28] T. Boiadjieva, G. Cappelletti, S. Ardizzone, S. Rondinini, and A. Vertova, "The role of surface electrification on the growth and structural features of titania nanoparticles," Physical Chemistry Chemical Physics, vol. 6, no. 13, pp. 3535-3539, 2004.

[29] M. A. Bhat, A. A. Dar, A. Amin, P. I. Rashid, and G. M. Rather, "Temperature dependence of transport and equilibrium properties of alkylpyridinium surfactants in aqueous solutions," Journal of Chemical Thermodynamics, vol. 39, no. 11, pp. 1500-1507, 2007.

[30] B. Simončič and J. Špan, "Thermodynamics of micellization of n-alkylpyridinium chlorides: a potentiometric study," Acta Chimica Slovenica, vol. 45, no. 2, pp. 143-152, 1998.

[31] K. Fujo and S. Ikeda, "Size of Micelles of 1-Dodecylpyridinium Chloride in Aqueous $\mathrm{NaCl}$ Solutions," Bulletin of the Chemical Society of Japan, vol. 65, pp. 1406-1410, 1992.

[32] J. J. Galán, A. González-Pérez, J. L. Del Castillo, and J. R. Rodríguez, "Thermal parameters associated to micellization of dodecylpyridinium bromide and chloride in aqueous solution," Journal of Thermal Analysis and Calorimetry, vol. 70, no. 1, pp. 229-234, 2002.

[33] A. N. Frumkin, "Die kapillarkurve der höheren fettsäuren und die zustandsgleichung der oberflächenschicht," Zeitschrift für Physikalische Chemie, vol. 166, pp. 466-484, 1925.

[34] R. H. Fowler and E. A. Guggenheim, Statistical Thermodynamics, Cambridge University Press, London, UK, 1965.

[35] A. de Keizer and L. G. J. Fokkink, "Specific adsorption of organic cations at the silver iodide-electrolyte interface," Colloids and Surfaces, vol. 51, pp. 323-337, 1990.

[36] S. Ardizzone, C. L. Bianchi, P. Quagliotto, and G. Viscardi, "Adsorption of cationic 'gemini' surfactants at the $\mathrm{TiO}_{2}$ /solution interface," Surface and Interface Analysis, vol. 34, no. 1, pp. 652-656, 2002.

[37] T. Mehrian, A. de Keizer, A. J. Korteweg, and J. Lyklema, "Thermodynamics of adsorption of dodecylpyridinium chloride on Na-kaolinite," Colloids and Surfaces A, vol. 73, pp. 133$143,1993$.

[38] R. H. Ottewill and M. C. Rastogi, "The stability of hydrophobic sols in the presence of surface-active agents-part 3. An examination by microelectrophoresis of the behaviour of silver iodide sols in the presence of cationic surface-active agents," Transactions of the Faraday Society, vol. 56, pp. 880-892, 1960.

[39] P. Somasundaran and D. W. Fuerstenau, "Mechanisms of alkyl sulfonate adsorption at the alumina-water interface," Journal of Physical Chemistry, vol. 70, no. 1, pp. 90-96, 1966.

[40] A. Fan, P. Somasundaran, and N. J. Turro, "Adsorption of alkyltrimethylammonium bromides on negatively charged alumina," Langmuir, vol. 13, no. 3, pp. 506-510, 1997.

[41] D. W. Fuerstenau and H. M. Jang, "On the nature of alkylsulfonate adsorption at the rutile/water interface," Langmuir, vol. 7, no. 12, pp. 3138-3143, 1991.

[42] M. J. Rosen, M. Dahanayake, and A. W. Cohen, "Relationship of structure to properties in surfactants. 11. surface and thermodynamic properties of N-dodecyl-pyridinium bromide and chloride," Colloids and Surfaces, vol. 5, no. 2, pp. 159-172, 1982.

[43] W. P. J. Ford, R. H. Ottewill, and H. C. Parreira, "Lightscattering studies on dodecylpyridinium halides," Journal of Colloid and Interface Science, vol. 21, no. 5, pp. 522-533, 1966.

[44] R. Zana, M. Benrraou, and R. Rueff, "Alkanediyl- $\alpha, \omega$ bis(dimethylalkylammonium bromide) surfactants. 1. Effect of the spacer chain length on the critical micelle concentration and micelle ionization degree," Langmuir, vol. 7, no. 6, pp. 1072-1075, 1991.
[45] R. Zana, H. Levy, D. Papoutsi, and G. Beinertdagger, "Micellization of two triquaternary ammonium surfactants in aqueous solution," Langmuir, vol. 11, no. 10, pp. 3694-3698, 1995.

[46] R. Zana, "Critical micellization concentration of surfactants in aqueous solution and free energy of micellization," Langmuir, vol. 12, no. 5, pp. 1208-1211, 1996.

[47] E. Alami, G. Beinert, P. Marie, and R. Zana, "Alkanediyl- $\alpha, \omega$ bis(dimethylalkylammonium bromide) surfactants. 3. Behavior at the air-water interface," Langmuir, vol. 9, no. 6, pp. 1465-1467, 1993.

[48] S. Manne, T. E. Schäffet, Q. Huo et al., "Gemini surfactants at solid-liquid interfaces: control of interfacial aggregate geometry," Langmuir, vol. 13, no. 24, pp. 6382-6387, 1997.

[49] M. Cao, X. Song, J. Wang, and Y. Wang, "Adsorption of hexyl- $\alpha, \omega$-bis(dodecyldimethylammonium bromide) gemini surfactant on silica and its effect on wettability," Journal of Colloid and Interface Science, vol. 300, no. 2, pp. 519-525, 2006.

[50] R. Atkin, V. S. J. Craig, E. J. Wanless, and S. Biggs, "Adsorption of 12-s-12 gemini surfactants at the silica-aqueous solution interface," Journal of Physical Chemistry B, vol. 107, no. 13, pp. 2978-2985, 2003.

[51] J. Yu, Y. Su, and B. Cheng, "Template-free fabrication and enhanced photocatalytic activity of hierarchical macro/mesoporous titania," Advanced Functional Materials, vol. 17, no. 12, pp. 1984-1990, 2007.

[52] J. Yu, L. Zhang, B. Cheng, and Y. Su, "Hydrothermal preparation and photocatalytic activity of hierarchically sponge-like macro-/mesoporous Titania," Journal of Physical Chemistry C, vol. 111, no. 28, pp. 10582-10589, 2007.

[53] J. Yu, W. Wang, B. Cheng, B. Huang, and X. Zhang, "Preparation and photocatalytic activity of multi-modally macro/mesoporous titania," Research on Chemical Intermediates, vol. 35, no. 6-7, pp. 653-665, 2009.

[54] H. Zhang and J. F. Banfield, "Thermodynamic analysis of phase stability of nanocrystalline titania," Journal of Materials Chemistry, vol. 8, pp. 2073-2076, 1998.

[55] M. Lazzeri, A. Vittadini, and A. Selloni, "Structure and energetics of stoichiometric $\mathrm{TiO}_{2}$ anatase surfaces," Physical Review B, vol. 63, no. 15, Article ID 155409, pp. 1-9, 2001.

[56] C.-C. Wang and J. Y. Ying, "Sol-gel synthesis and hydrothermal processing of anatase and rutile titania nanocrystals," Chemistry of Materials, vol. 11, no. 11, pp. 3113-3120, 1999.

[57] G. Cappelletti, C. Ricci, S. Ardizzone, C. Parola, and A. Anedda, "Aged titania nanoparticles: the simultaneous control of local and long-range properties," Journal of Physical Chemistry $B$, vol. 109, no. 10, pp. 4448-4454, 2005. 

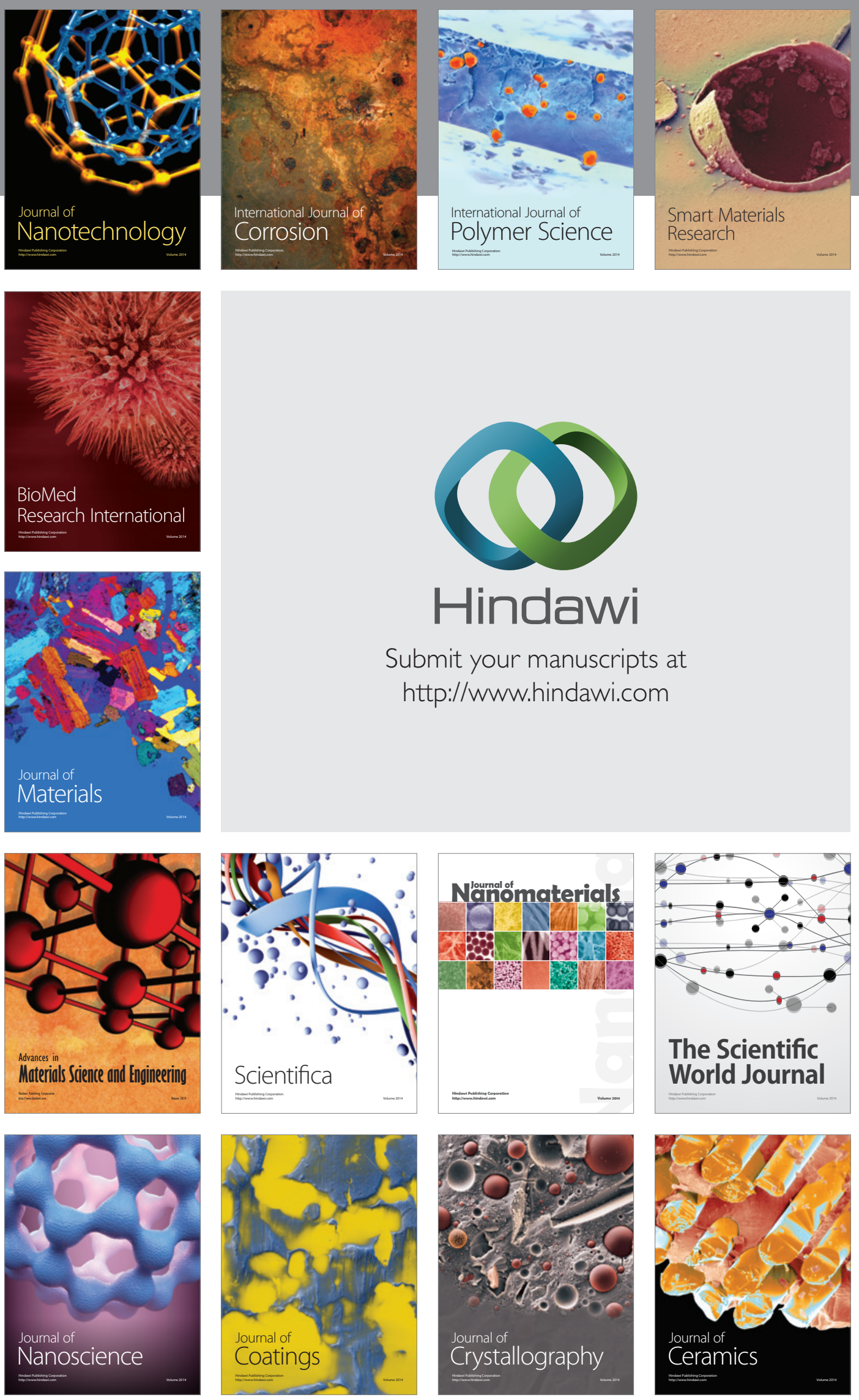

The Scientific World Journal

Submit your manuscripts at

http://www.hindawi.com

\section{World Journal}

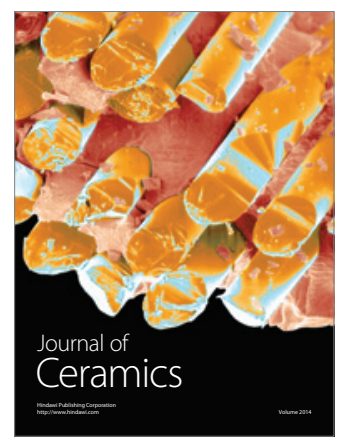

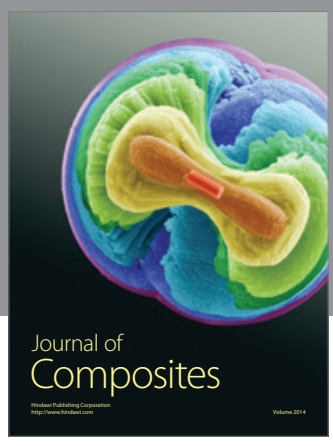
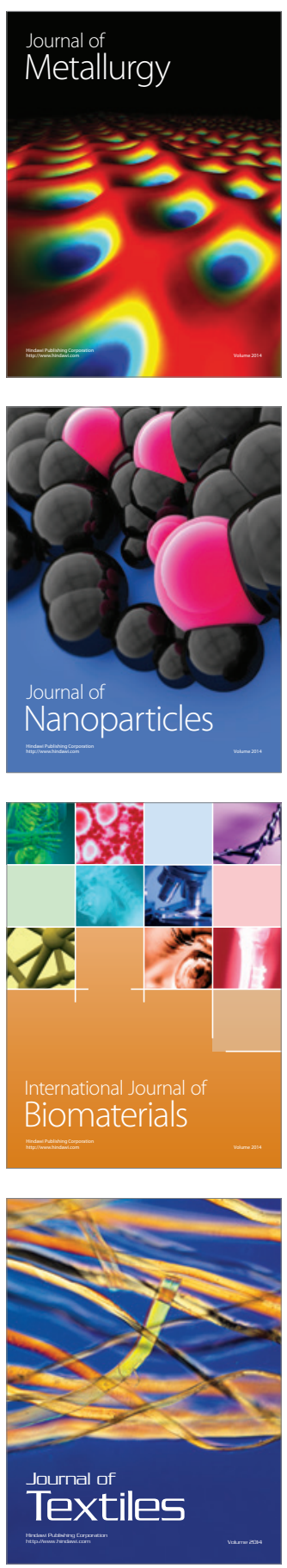